\title{
Systemic Tumor Seeding Secondary to Peritonitis due to the Immediate Postoperative Intravesical Instillation of Pirarubicin After Intravesical Explosion Induced Bladder Perforation During Bipolar Transurethral Resection of Bladder Tumors
}

\author{
Kenji Ohgaki ${ }^{\mathrm{a}, \mathrm{b}}$, Masatoshi Sakamoto ${ }^{\mathrm{a}}$
}

\begin{abstract}
An 82-year-old man with multiple nonmuscle-invasive bladder cancer underwent transurethral resection (TUR) of bladder tumor by using the transurethral resection in saline (TURis) system. Intravesical explosion occurred during the resection of a tumor of the bladder dome, but no bladder perforation was detected. Immediate postoperative intravesical instillation of $30 \mathrm{mg}$ pirarubicin in $30 \mathrm{~mL}$ saline was administered after the TURis. After this procedure, the patient complained of severe lower abdominal pain. A computed tomography (CT) scan after cystography revealed a collection of fluid in the abdominal cavity with signs of urine leakage. It was determined that instillation of pirarubicin led to leakage outside the bladder through a near perforation on the bladder that occurred during TURis. An indwelling 20 Fr Foley catheter was placed for 8 days. His condition improved without additional surgery. After the patient was discharged, gross hematuria appeared. A residual tumor was confirmed near the perforation area. Therefore, 3.5 months after the first TURis, he underwent a second TURis. Dyspnea appeared 1 month after the second TURis. Positron emission tomography and computed tomography (PET/CT) imaging revealed systemic tumor seeding. Five months after surgery, the patient died because of acute respiratory failure.
\end{abstract}

Keywords: Bipolar transurethral resection of bladder tumors; Intravesical explosion; Immediate postoperative intravesical instillation of pirarubicin; Systemic tumor seeding

\section{Introduction}

Tumor cell implantation during transurethral resection (TUR)

Manuscript submitted March 5, 2019, accepted April 3, 2019

a Department of Urology, Asao General Hospital, Kanagawa, Japan ${ }^{b}$ Corresponding Author: Kenji Ohgaki, Department of Urology, Asao General Hospital, 6 Chome-25-1 Kamiasao Asao Ward, Kawasaki, Kanagawa 2150021, Japan. Email: kogaki2018@gmail.com

doi: https://doi.org/10.14740/jmc3277 is an important factor for tumor recurrence. Therefore, immediate postoperative intravesical treatment with a chemotherapeutic agent after TUR for nonmuscle-invasive bladder cancer is the standard of care $[1,2]$. Compared with adriamycin, pirarubicin is more rapidly taken up by bladder tumor cells and is able to exert an antitumor effect on a bladder tumor in a shorter time $[3,4]$. However, this procedure is not always harmless. When a bladder perforation or near perforation occurs during or after TUR, the immediate instillation of a chemotherapeutic agent may cause extravesical leakage such as leakage into the retroperitoneal or intraperitoneal cavity $[5,6]$. We report a case wherein the patient died because of acute respiratory failure associated with systemic tumor seeding after the immediate postoperative instillation of pirarubicin through a bladder injury caused by intravesical explosion.

\section{Case Report}

An 82-year-old man with type 2 diabetes presented with multiple papillary tumors on the posterior bladder wall and in the dome of the bladder (Fig. 1). He underwent a TUR under general anesthesia. The transurethral resection in saline (TURis) system was used for TUR with a 24 Fr sheath. The cutting and coagulation powers were set to 280 watts and 100 watts, respectively. During the resection of papillary tumors, an intravesical explosion of the air bubbles at the bladder dome occurred with little sound. However, bladder perforation was not detected. The surgery time was $59 \mathrm{~min}$ and the amount of bleeding was very minimal. A three-way 20 Fr Foley catheter was placed inside the bladder. An immediate postoperative instillation of $30 \mathrm{mg}$ pirarubicin in $30 \mathrm{~mL}$ saline was maintained in the bladder for $5 \mathrm{~min}$. He received continuous saline bladder irrigation after the TURis. When he returned to the general hospital room after surgery, he complained of severe abdominal pain. On examination, he was afebrile $\left(36.0^{\circ} \mathrm{C}\right)$ and had a blood pressure of $117 / 50 \mathrm{~mm} \mathrm{Hg}$ and a pulse rate of 53 beats per minute. Oxygen saturation was $100 \%$ under $5 \mathrm{~L}$ of oxygen. He did not have gross hematuria under manual bladder irrigation. Bladder injury was suspected because a noncontrast abdominopelvic 


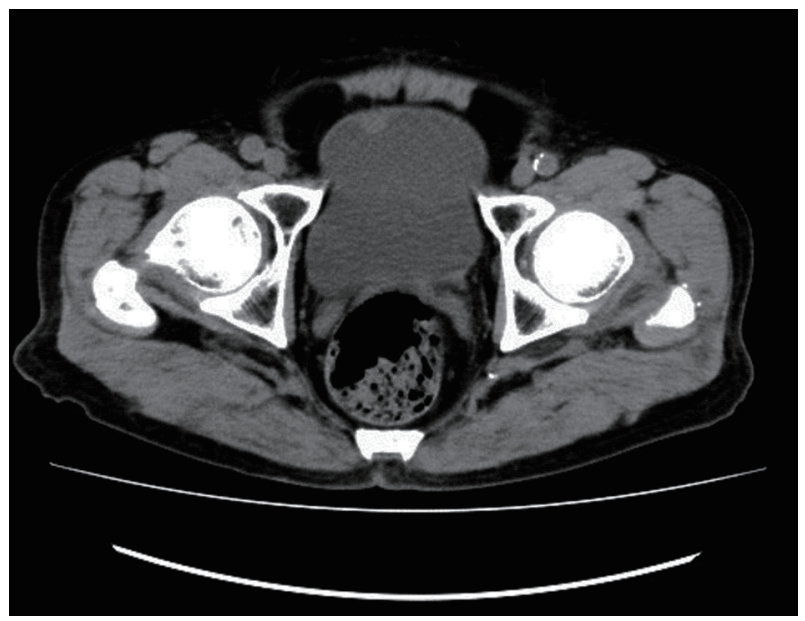

Figure 1. Noncontrast computed tomography scan at the urinary bladder level shows a mass in the bladder dome.

computed tomography (CT) scan revealed a collection of fluid in the abdominal cavity. A second CT cystogram showed extravasation of the contrast agent from the dome of the bladder (Fig. 2). He had been scheduled for an exploratory laparotomy; therefore, he was moved again to the operating room. However, his abdominal pain had disappeared. Ultrasonography revealed the loss of fluid from the abdominal cavity, and his laparotomy was consequently postponed.

One day after surgery, a third noncontrast $\mathrm{CT}$ scan revealed loss of fluid from the abdominal cavity, and his symptom remained resolved. On examination, he was afebrile $\left(36.5^{\circ} \mathrm{C}\right)$ and had a blood pressure of 102/56 $\mathrm{mm} \mathrm{Hg}$ and a pulse rate of 63 beats per minute. Oxygen saturation was $95 \%$ in room air. $\mathrm{He}$ was diagnosed as having an extensive intraperitoneal bladder injury caused by intravesical explosion during TURis after the immediate postoperative intravesical instillation of a single dose of pirarubicin. Eight days after surgery, a cystogram showed no extravasation of the contrast agent (Fig. 3). Nine days after sur-

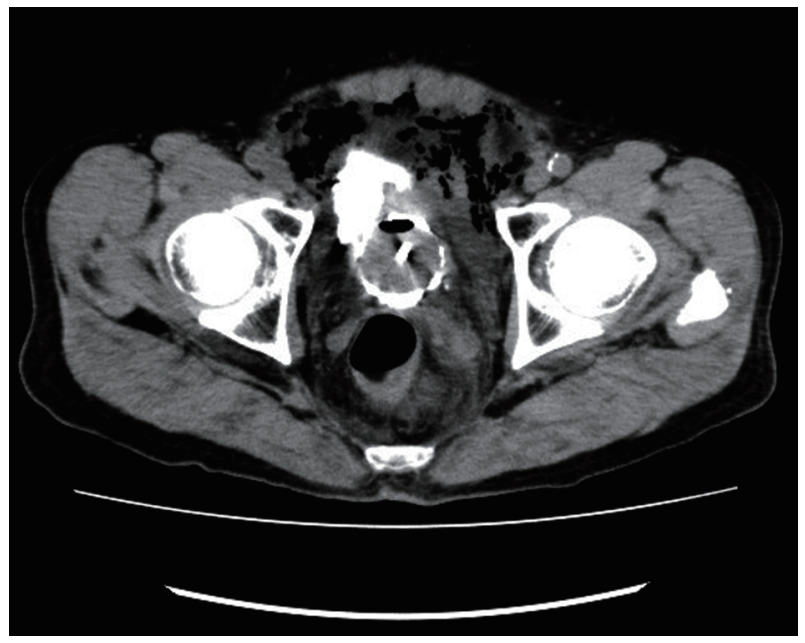

Figure 2. A computed tomography cystogram at the urinary bladder level shows extravasation of the contrast agent from the bladder dome immediately after surgery.

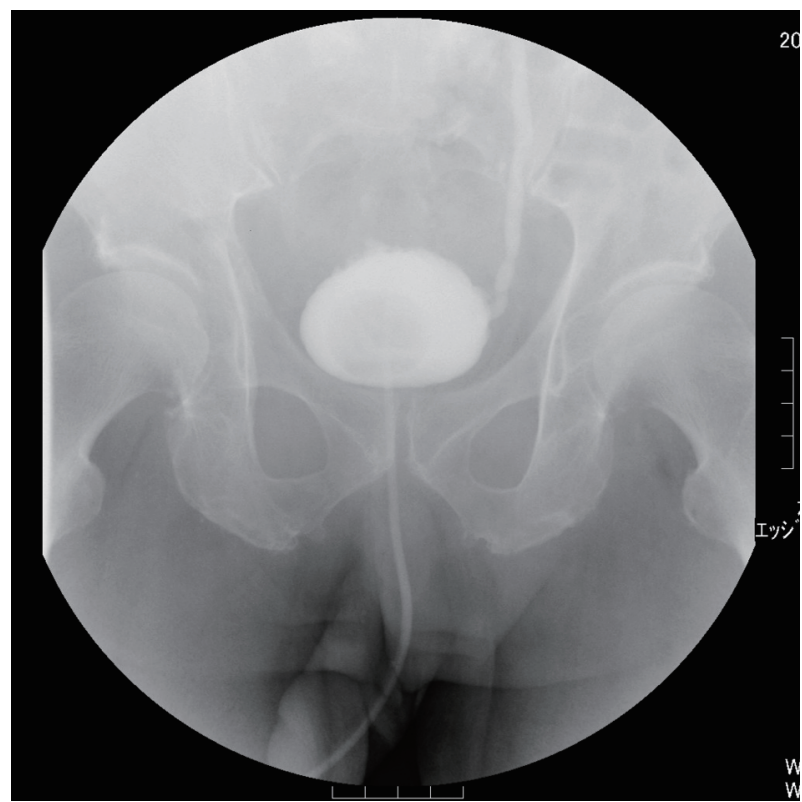

Figure 3. Eight days after surgery, a conventional cystogram shows no extravasation of the contrast agent from the bladder dome.

gery, the patient was discharged in good condition because he could urinate after the removal of the urethral catheter. Histologic examination confirmed G2 pTa urothelial carcinoma.

After discharge, he received $30 \mathrm{mg}$ pirarubicin intravesical instillation therapy four times. Three months after surgery, he underwent cystoscopy because of the recurrence of gross hematuria. A hemorrhagic ulcerous tumor was found near the perforated area. Therefore, he underwent a second TURis 3.5 months after the first TURis. Pathological findings were urothelial carcinoma (G2, pT1).

After discharge, gross hematuria also recurred. Therefore, he underwent bladder magnetic resonance imaging (MRI). Bladder wall invasion in the bladder dome was suspected (Fig. 4). Dyspnea and chest pain appeared 4.5 months after the first TURis. Positron emission tomography and computed tomography (PET/CT) showed abnormal accumulation in the right supe-

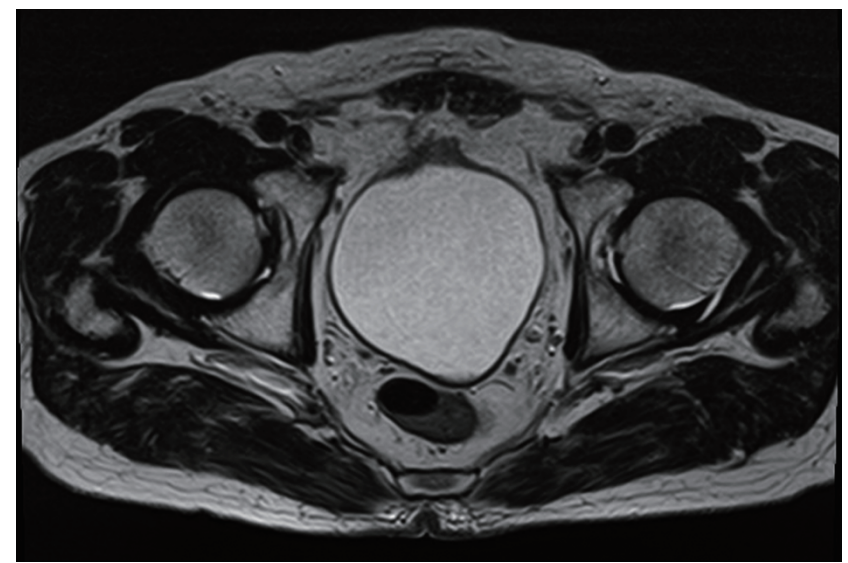

Figure 4. Noncontrast magnetic resonance imaging at the urinary bladder level shows the thick wall of the bladder dome. 


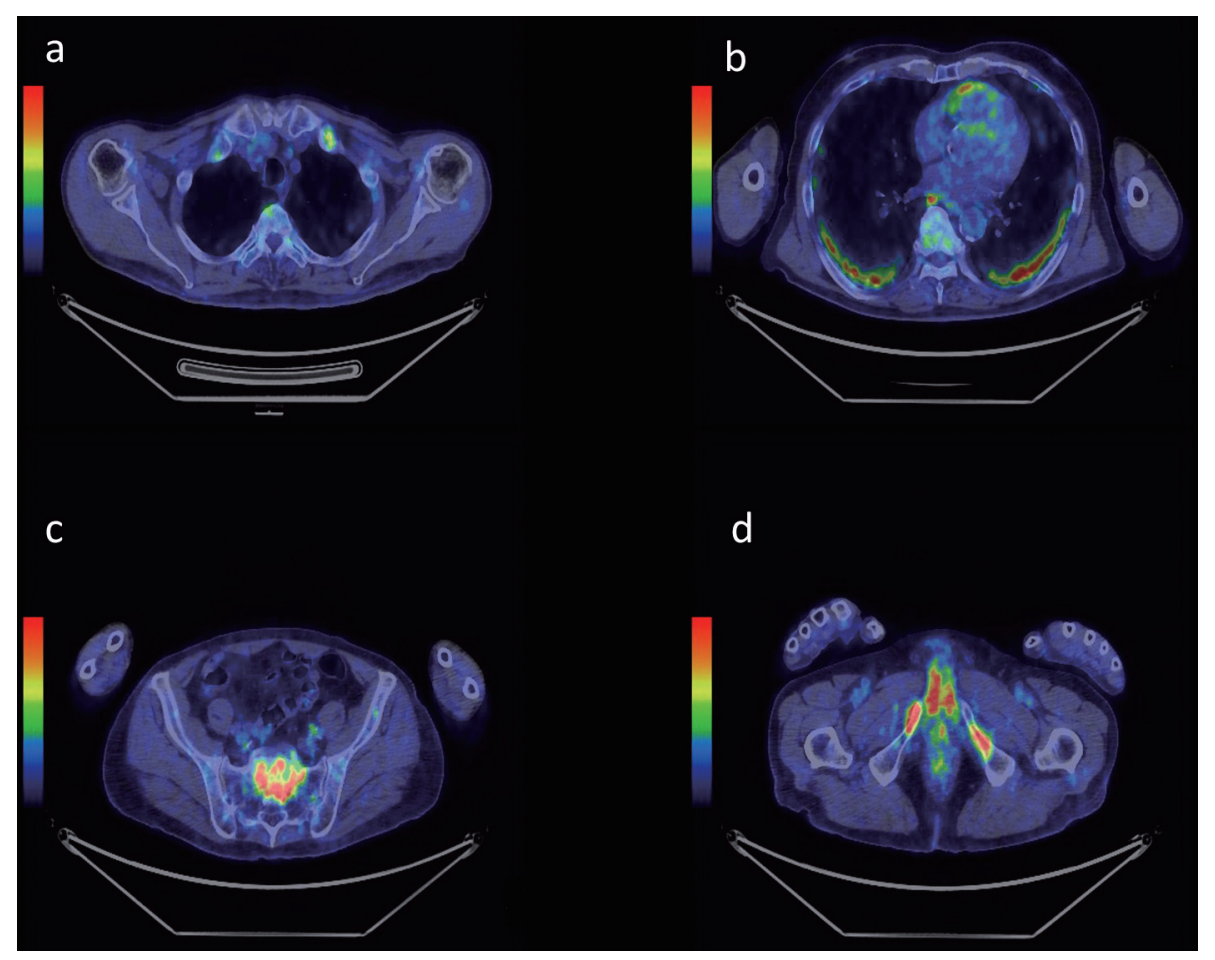

Figure 5. Positron emission tomography and computed tomography imaging shows the uptake in (a) the left rib and thoracic spine, (b) the bilateral lungs, (c) the sacrum and (d) the bilateral pubic bones.

rior supraclavicular fossa, left rib (Fig. 5a), bilateral lungs (Fig. $5 b$ ), right pleura, left internal iliac lymph node, lumbar spine, sacrum (Fig. 5c), bilateral pubic bone (Fig. 5d), and right femur.

Five months after his first surgery, he was hospitalized because of enhanced dyspnea. On examination, he was afebrile $\left(36.7^{\circ} \mathrm{C}\right.$ ) and had a blood pressure of $108 / 52 \mathrm{~mm} \mathrm{Hg}$ and a pulse rate of 86 beats per minute. Oxygen saturation was $91 \%$ in room air. The next day, he died because of acute respiratory failure.

\section{Discussion}

A small intravesical explosion, which usually manifests as a "pop" sound, would be experienced during transurethral surgery. Damage to the bladder because of a major intravesical explosion can range from small mucosal tears to bladder rupture, which can be intraperitoneal or extraperitoneal. Intravesical explosions occur because of the production of explosive gases during use of diathermy on human tissues. Hydrogen is formed by the pyrolysis of bladder tissue caused by diathermy, and it collects under the dome of the bladder. Hydrogen alone is not explosive; it only becomes explosive when admixed with oxygen. Oxygen is not produced in sufficient quantity during diathermy to cause explosions but it can enter the bladder from the atmosphere during endoscopic procedures. Once hydrogen combines with oxygen, it becomes explosive and can be ignited by spark generated during electrosurgery [7].

In this patient, a small explosion occurred while resecting the dome tumor, but the surgeon did not care. A near perfora- tion was observed in the bladder, but a clear perforation was not detected with cystoscopy. Therefore, pirarubicin was immediately instilled postoperatively. Symptoms of peritoneal irritation such as abdominal colic and abdominal distension were severe because pirarubicin, which had been injected immediately after surgery, had leaked out of the bladder. Bladder extravasation was confirmed by cystogram CT examination and the patient was diagnosed as having peritonitis caused by bladder perforation. Repair surgery for bladder perforation was urgently scheduled. However, the patient's symptoms were relieved by analgesics and catheter placement; therefore, his operation was postponed.

It is important that physicians recognize abdominal symptoms such as peritonitis and ileus in patients who have received intravesical chemotherapy immediately after TUR of bladder tumor (TURBT). Transurethral surgery is often performed under spinal anesthesia to help confer good postoperative analgesia and to reduce the stress response to surgery. The early detection of hyponatremia associated with TUR syndrome is facilitated in a conscious patient [8]. If transurethral surgery is continued without the surgeon's being aware of a bladder perforation in a patient during general anesthesia, then hyponatremia due to TUR syndrome may be aggravated because the patient is unconscious. However, the TURis procedure is less likely to cause TUR syndrome [9]. Poor postoperative analgesia after general anesthesia may have aided the appearance of prominent lower abdominal pain due to bladder perforation in the patient. This factor may have led to the early detection postoperatively of the bladder perforation.

It has been reported that bladder perforation during 
TURBT does not substantially increase the risk of extravesical tumor seeding, and that surgical repair of a bladder perforation during TURBT somewhat increases the risk of extravesical tumor cell recurrence and negatively affects a patient's prognosis [10]. Owing to the low rate of tumor seeding resulting from bladder perforation during TURBT, extravesical seeding of a tumor may not have occurred during TURis in our patient. In the TURis system, fine air bubbles generated by plasma discharge will accumulate in the bladder dome [11]. Bubbles make it difficult to recognize a top dome tumor. The accumulated air bubbles will need to be removed to reduce the possibility of intravesical explosion [12]. Pirarubicin is more rapidly taken up by bladder tumor cells and can exert an antitumor effect on a bladder tumor in a shorter time. Therefore, immediate postoperative intravesical treatment with a chemotherapeutic agent after TUR for nonmuscle-invasive bladder cancer is the standard of care [1-4]. Systemic dissemination would unlikely occur in the early postoperative period because of the antitumor effect by the immediate postoperative intravesical instillation of pirarubicin. The pathological diagnosis confirmed G2, pTa urothelian carcinoma. However, the patient achieved systemic dissemination early after TURis. Systemic dissemination may result from the extravasation of tumor cells that may have remained, along with pirarubicin, in the bladder immediately after TURis. When bladder perforation is suspected during TUR, an immediate postoperative intravesical instillation of pirarubicin should be avoided.

\section{Conclusions}

Intravesival explosion is a rare complication, but it can lead to a serious condition. Urologists should pay attention to intravesical explosion, and must remove air bubbles during the resection of tumors in the bladder dome. For most patients with superficial bladder cancer, the benefits of immediate intravesical chemotherapy after TUR exceed the risk of serious complications. However, an immediate postoperative instillation has to be avoided when there is an obvious or suspect bladder perforation. Low-grade superficial bladder cancer has the possibility of tumor seeding. Therefore, it should be observed strictly after perforation.

\section{Acknowledgments}

None to declare.

\section{Financial Disclosure}

No funding was received for the publication of this study.

\section{Conflict of Interest}

The authors declare that there is no conflict of interest associated with the work described in the manuscript.

\section{Informed Consent}

The patient has provided permission for us to publish the features of his case, and the identity of the patient has been protected.

\section{Author Contributions}

All authors were involved in patient care. Co-authors helped to write or edit the manuscript. All authors read and approved the final manuscript.

\section{Ethical Approval}

This study was approved by the appropriate ethics committee and therefore was performed in accordance with the ethical standards laid down in the 1964 Declaration of Helsinki and its later amendments.

\section{References}

1. Kang M, Jeong CW, Kwak C, Kim HH, Ku JH. Single, immediate postoperative instillation of chemotherapy in non-muscle invasive bladder cancer: a systematic review and network meta-analysis of randomized clinical trials using different drugs. Oncotarget. 2016;7(29):4547945488 .

2. Sylvester RJ, Oosterlinck W, Holmang S, Sydes MR, Birtle A, Gudjonsson S, De Nunzio C, et al. Systematic review and individual patient data meta-analysis of randomized trials comparing a single immediate instillation of chemotherapy after transurethral resection with transurethral resection alone in patients with stage pTa-pT1 urothelial carcinoma of the bladder: which patients benefit from the instillation? Eur Urol. 2016;69(2):231-244.

3. Akaza H, Niijima T, Hisamatsu T, Fujigaki M. Comparative investigation on use of (2"R)-4'-O-tetrahydropyranyladriamycin and adriamycin as intravesical chemotherapy for superficial bladder tumors. Urology. 1988;32(2):141145.

4. Okamura K, Ono Y, Kinukawa T, Matsuura O, Yamada $\mathrm{S}$, Ando T, Fukatsu T, et al. Randomized study of single early instillation of (2"R)-4'-O-tetrahydropyranyl-doxorubicin for a single superficial bladder carcinoma. Cancer. 2002;94(9):2363-2368.

5. Oddens JR, van der Meijden AP, Sylvester R. One immediate postoperative instillation of chemotherapy in low risk Ta, T1 bladder cancer patients. Is it always safe? Eur Urol. 2004;46(3):336-338.

6. Lim D, Izawa JI, Middlebrook P, Chin JL. Bladder perforation after immediate postoperative intravesical instillation of mitomycin C. Can Urol Assoc J. 2010;4(1):E1-3.

7. Khan A, Masood J, Ghei M, Kasmani Z, Ball AJ, Miller R. Intravesical explosions during transurethral endoscopic procedures. Int Urol Nephrol. 2007;39(1):179-183. 
8. McGowan-Smyth S, Vasdev N, Gowrie-Mohan S. Spinal anesthesia facilitates the early recognition of TUR syndrome. Curr Urol. 2016;9(2):57-61.

9. Michielsen DP, Coomans D, Braeckman JG, Umbrain $\mathrm{V}$. Bipolar transurethral resection in saline: the solution to avoid hyponatraemia and transurethral resection syndrome. Scand J Urol Nephrol. 2010;44(4):228-235.

10. Golan S, Baniel J, Lask D, Livne PM, Yossepowitch O. Transurethral resection of bladder tumour complicated by perforation requiring open surgical repair - clini- cal characteristics and oncological outcomes. BJU Int. 2011;107(7):1065-1068.

11. Kass-iliyya A, Warren K. Beware the large air bubble: a case report of bladder explosion during transurethral resection of bladder tumour. Journal of Urology Forecast. 2018;1(1):1-2.

12. Lee CC, Chang HK, Chen M, Hsu JM. Intravesical explosion resulting in bladder rupture during transurethral resection of bladder tumors. International Journal of Gerontology. 2014;8(4):230-231. 